\title{
Decrease in the Kainate-Induced Wet Dog Shake Behavior in Genetically Epilepsy-Prone Rats: Possible Involvement of an Impaired Synaptic Transmission to the 5-HT ${ }_{2 \mathrm{~A}}$ Receptor
}

\author{
Eun-Joo Shin ${ }^{1, \dagger}$, Ji Hoon Jeong ${ }^{2, \dagger}$, Yoon Hee Chung ${ }^{3}$, Tae-Woo Kim ${ }^{1}$, Chan Young Shin ${ }^{4}$, Won-Ki Kim ${ }^{5}$, \\ Kwang-Ho $\mathrm{Ko}^{6}$, and Hyoung-Chun $\mathrm{Kim}^{1, *}$ \\ ${ }^{I}$ Neuropsychopharmacology and Toxicology Program, College of Pharmacy, Kangwon National University, \\ Chunchon 200-701, Republic of Korea \\ Departments of ${ }^{2}$ Pharmacology and ${ }^{3}$ Anatomy, College of Medicine, Chung-Ang University, Seoul 156-756, Republic of Korea \\ ${ }^{4}$ Department of Pharmacology, School of Medicine, Konkuk University, Seoul 143-701, Republic of Korea \\ ${ }^{5}$ Department of Neuroscience, School of Medicine, Korea University, Seoul 136-701, Republic of Korea \\ ${ }^{6}$ Department of Pharmacology, College of Pharmacy, Seoul National University, Seoul 151-742, Republic of Korea
}

Received January 15, 2009; Accepted May 6, 2009

\begin{abstract}
Genetically epilepsy-prone rats (GEPR-9s) were derived from Sprague-Dawley rats (SD). The number of kainate-induced wet dog shake behavior (WDS) responses was found to decrease significantly in GEPR-9s compared to SD. WDS responses were potentiated by 5hydroxytryptophan or 2,5-dimethoxy-4-iodoamphetamine and antagonized by ritanserin. The antagonizing effect of ritanserin on WDS latency was more evident in GEPR-9s than in SD, and hippocampal expression of activity-regulated cytoskeleton-associated protein paralleled the severity of WDS. The results suggest that downstream serotonergic synaptic activation is less pronounced in GEPR-9s than in SD and that the serotonergic agent may directly activate postsynaptic 5-HT2A receptors in both strains.
\end{abstract}

Keywords: kainic acid, genetically epilepsy-prone rat, wet dog shake

Genetically epilepsy-prone rats (GEPRs) are models of generalized tonic/clonic epilepsy and have been used to study the basic mechanisms of human epilepsy. The physiological and biochemical traits found in GEPRs that are not found in the parent strain (Sprague-Dawley rats, $\mathrm{SD}$ ) are presumed to be abnormal (1) and to include dysfunctional noradrenergic, serotonergic, GABAergic, dopaminergic, and/or glutamatergic neurons $(1,2)$.

Systemic administration of kainate (KA) produces various motor signs, including convulsive seizures. Wet dog shake behavior (WDS), a pre-phase for a KAinduced convulsive seizure, can be identified as a reflexive shudder of the head, neck, and trunk. Previous research has indicated that the WDS can be mediated by selective activation of the $5-\mathrm{HT}_{2 \mathrm{~A}}$ receptor (3). Thus,

†First two authors equally contributed to this work.

*Corresponding author. kimhe@kangwon.ac.kr

Published online in J-STAGE

doi: $10.1254 /$ jphs.09015SC
WDS appears to be a useful, reliable, and non-invasive gauge of alterations in endogenous $5-\mathrm{HT}_{2 \mathrm{~A}}-$ receptor activity (4).

Activity-regulated, cytoskeleton-associated protein (Arc) has been recognized as an immediate-early effector gene regulated by synaptic activity (5), and synaptic activation has been shown to cause newly synthesized Arc protein to localize selectively in activated post-synaptic sites in dendrites (6). Selective serotonin reuptake inhibitors (SSRIs) elevate synaptic serotonin levels, and this may contribute to up-regulating Arc gene expression (7).

In the present study, we examined whether KA seizure intensity coincided with WDS in both GEPR-9s and $\operatorname{SD}(1,8)$, whether WDS was regulated by the serotonergic system, and whether modulation of serotonergic function altered Arc expression in KA-treated GEPR-9s and SD.

All animals were treated in accordance with the NIH Guide for the Humane Care and Use of Laboratory 
Animals. Male SD (controls) and GEPR-9s were kept on a 12:12 h light:dark cycle and fed ad libitum. KA; 5-hydroxytryptophan (HTP), a serotonin precursor; ritanserin (RI), a serotonin $5-\mathrm{HT}_{2 \mathrm{~A}}-$ receptor antagonist; and 2,5-dimethoxy-4-iodoamphetamine (DOI), a serotonin 5- $\mathrm{HT}_{2 \mathrm{~A}}-$ receptor agonist were purchased from Sigma Chemical Company (St. Louis, MO, USA). HTP ( $75 \mathrm{mg} / \mathrm{kg}$, i.p.) or DOI $(0.3 \mathrm{mg} / \mathrm{kg}$, s.c.) was injected $15 \mathrm{~min}$ before KA $(8 \mathrm{mg} / \mathrm{kg}$, i.p. $)$ treatment, and RI $(0.5 \mathrm{mg} / \mathrm{kg}$, i.p.) was given $45 \mathrm{~min}$ before KA treatment.

Using an automated video tracking system (Noldus Information Technology, Wageningen, Netherlands), WDS and convulsive seizures were recorded during a 4-h period following the KA challenge. Seizure scores were rated according to the scale of Racine (9); animals were scored after having had three consecutive seizures at each stage.

Animals were sacrificed 1.5 (for WDS) and $4 \mathrm{~h}$ (for seizure) after KA administration. Brains were rapidly removed, and hippocampi were dissected and then stored at $-80^{\circ} \mathrm{C}$. Western blot analysis was performed as described previously (2). Each blot was incubated with a primary antibody against $\operatorname{Arc}(1: 1000$; Santa Cruz Biotechnology, Inc., Santa Cruz, CA, USA) or $\beta$-actin (1:5000; Sigma). Relative intensities of the bands were quantified by PhotoCapt MW (version 10.01 for Windows; Vilber Lourmat, Marne la Vallée, France).

Statistical analyses were performed using one-way analysis of variance (ANOVA). Significance was assessed by the post-hoc Fisher's PLSD test. A $P$ value less than 0.05 was deemed to be statistically significant.

To test the effects of KA on WDS and seizure activity, we injected both rat strains with $8 \mathrm{mg} / \mathrm{kg} \mathrm{KA}$ because GEPR-9s that received $10 \mathrm{mg} / \mathrm{kg}$ KA died within $6 \mathrm{~h}$ in a pilot experiment. Neither spontaneous seizure activity nor any abnormal behavior was observed in GEPR-9s under our experimental conditions.

Figure 1 shows KA-induced WDS and hippocampal Arc expression in both strains. WDS after KA treatment appeared with an earlier latency in SD $(18.5 \pm 2.6 \mathrm{~min})$ $(P<0.05)$ than in GEPR-9s $(25.2 \pm 3.7 \mathrm{~min})$. RI significantly extended $(P<0.05)$ WDS latencies in both strains; HTP and DOI significantly shortened $(P<0.05)$ WDS latencies in both strains. RI significantly counteracted the shortening effect mediated by HTP or DOI. The reverse effect of RI on WDS latency was more pronounced in GEPR-9s treated with HTP $(P<0.05$ in $\mathrm{SD}, P<0.01$ in GEPR-9s $)$ or DOI $(P<0.01$ in $\mathrm{SD}$, $P<0.001$ in GEPR-9s) than in SD (Fig. 1A).

The number of WDS after KA treatment in SD $(167.5 \pm 22)$ was significantly higher $(P<0.01)$ than in GEPR-9s $(71.5 \pm 11.8)$. Although no WDS was observed in the absence of KA, a mild to moderate level

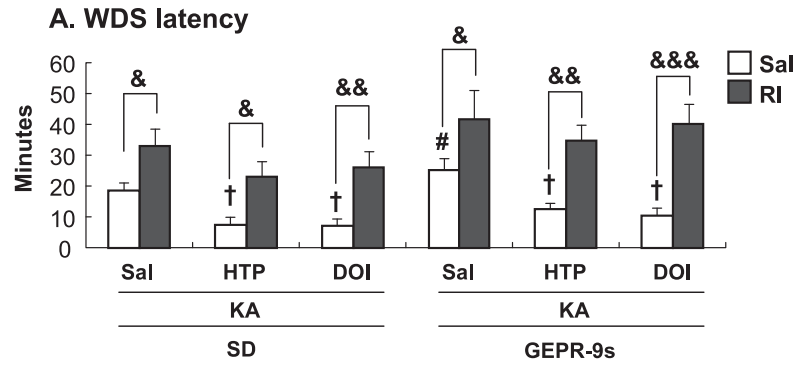

B. WDS number

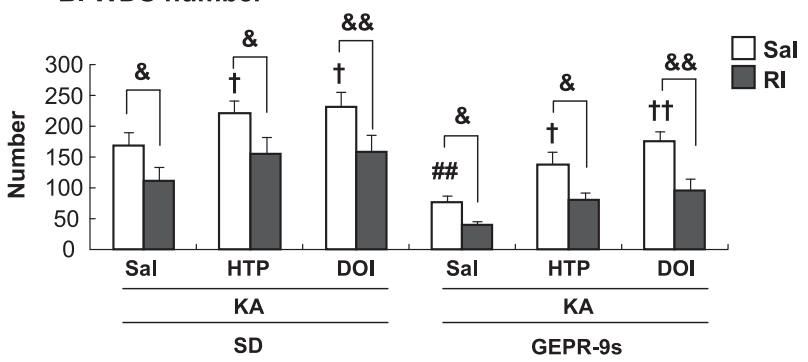

C. Arc-IR

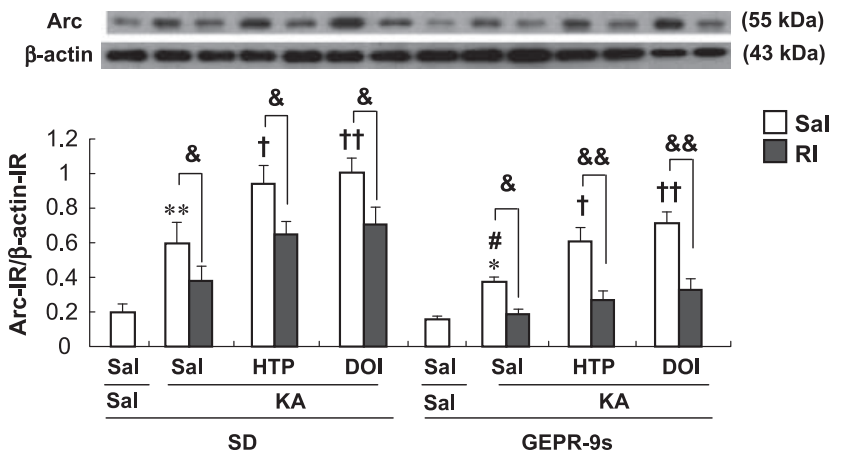

Fig. 1. Effects of ritanserin (RI, $0.5 \mathrm{mg} / \mathrm{kg}$, i.p.) on 5-hydroxytryptophan (HTP, $75 \mathrm{mg} / \mathrm{kg}$, i.p.)- or 2,5-dimethoxy-4-iodoamphetamine (DOI, $0.3 \mathrm{mg} / \mathrm{kg}$, s.c.)-mediated pharmacological responses to $\mathrm{KA}(8 \mathrm{mg} / \mathrm{kg}$, i.p.)-induced wet dog shake (WDS) behavior [latency (A), number (B)] and hippocampal Arc-IR (C) $1.5 \mathrm{~h}$ after KA in SD rats and GEPR-9s. Sal = saline treatment. Arc-IR = Activityregulated, cytoskeleton-associated protein immunoreactivity. Each value is the mean \pm S.E.M. of 8 animals for wet dog shake behaviors and 5 animals for Arc expression. ${ }^{*} P<0.05,{ }^{* *} P<0.01$ vs. corresponding $\mathrm{Sal}+\mathrm{Sal} ;{ }^{\dagger} P<0.05,{ }^{\dagger} P<0.01 \quad$ vs. corresponding $\mathrm{Sal}+\mathrm{KA}$; ${ }^{\#} P<0.05,{ }^{\#} P<0.01$ vs. Sal + KA-treated SD; ${ }^{\&} P<0.05$, ${ }^{\&} \&<0.01$, or ${ }^{\& \& \&} P<0.001$, inter-group (one-way ANOVA followed by Fisher's PLSD test).

of activity-regulated, cytoskeleton-associated protein immunoreactivity (Arc-IR) was observed in the hippocampus in both strains. Treatment with RI significantly decreased WDS number $(P<0.05)$ and Arc-IR $(P<0.05)$ in both strains. In contrast, treatment with HTP significantly increased WDS number $(P<0.05)$ and Arc-IR $(P<0.05)$ in both strains. DOI significantly increased WDS number and Arc-IR in SD by $49 \%(P<0.05)$ and 68\% $(P<0.01)$, respectively, whereas DOI markedly increased WDS number by $132 \%(P<0.01)$ and Arc-IR 
by $91 \%(P<0.01)$ in GEPR-9. In SD, RI significantly decreased the HTP- or DOI-mediated increase in WDS number by $33 \%(P<0.05)$ or $35 \%(P<0.05)$, respectively, and Arc-IR by $31 \%(P<0.05)$ or $29 \%(P<0.05)$. A more prominent effect of RI was noted in GEPR-9s; RI reversed the HTP- or DOI-mediated increase in WDS number by $41 \%(P<0.05)$ or $48 \%(P<0.01)$, respectively, and Arc-IR by $55 \%(P<0.01)$ or $54 \%(P<0.01)$ (Fig. 1 : B and C).

Figure 2 shows the effects of RI on the HTP- or DOImediated action against KA-induced seizures in both strains. KA treatment led to robust behavioral seizures in both strains, lasting $4-5 \mathrm{~h}$. The seizures were more severe in GEPR-9s (both seizure latency and seizure score, $P<0.05$ ) than in SD (Fig. 2: A and B). Furthermore, exposure to RI alone, HTP alone, RI plus HTP, DOI alone, or RI plus DOI did not significantly alter seizure activity, seizure latency, or mortality after KA treatment (Fig. 2: A and B). KA-induced increases in Arc-IR $(P<0.01$ vs. saline plus saline in SD or GEPR-9s) were observed in both strains. However, RI alone, HTP alone, RI plus HTP, DOI alone, or RI plus DOI did not significantly affect Arc-IR induced by KA (Fig. 2C).

The present study showed a higher sensitivity to KAinduced seizures in GEPR-9s than in SD. Although the decrease in WDS latency and increase in WDS number may depend on seizure activity (9), our results showed that the change in WDS responses (i.e., latency and number) induced by KA was less pronounced in GEPR9 s than in SD, suggesting a divergence in epileptogenic mechanisms in the rat strains. We observed that exposure to HTP or DOI before KA treatment increased WDS number (while reducing WDS latency), and RI significantly reversed the WDS responses mediated by HTP or DOI. These findings suggest that $5-\mathrm{HT}_{2 \mathrm{~A}}$ receptor-mediated signaling did not appear to be impaired and rather that serotonergic synaptic transmission downstream of KA-induced neuronal excitation may be impaired.

Arc is recognized to play a key role in an experimental model of synaptic activation and increases in Arc expression have been, at least in part, linked to neuronal adaptation (6). Arc, however, is not the only molecule responsible for modulating neuronal networks; thus, changes in Arc expression alone cannot bring about the final changes in network plasticity.

Our data are consistent with those of Li et al. (10), who found that Arc protein expression is most significantly increased at $2-4 \mathrm{~h}$ post-KA in the hippocampus. However, Arc mRNA expression in the hippocampus was not significantly altered by a serotonergic agent alone (11). Thus, we cannot rule out the possibility that a serotonergic agent may indirectly modulate
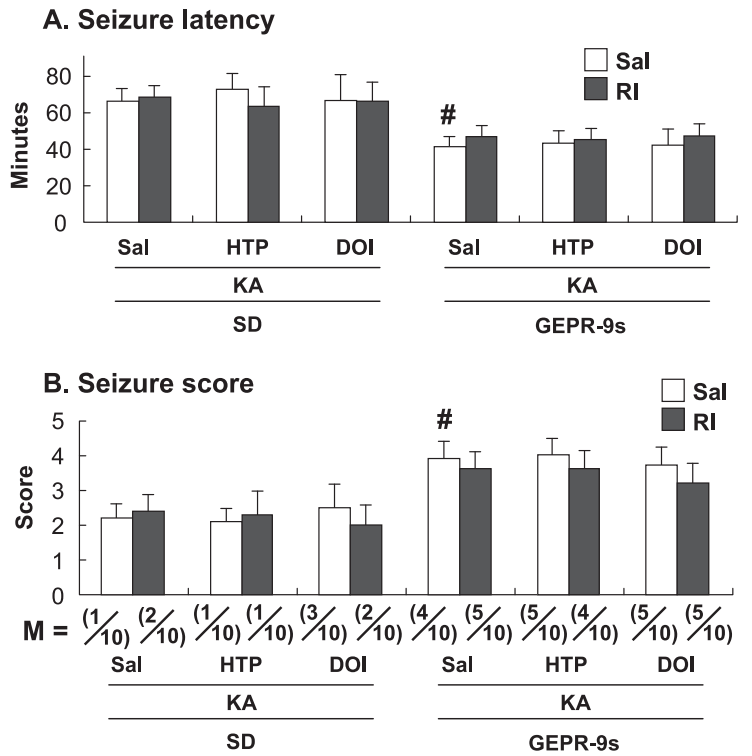

C. Arc-IR

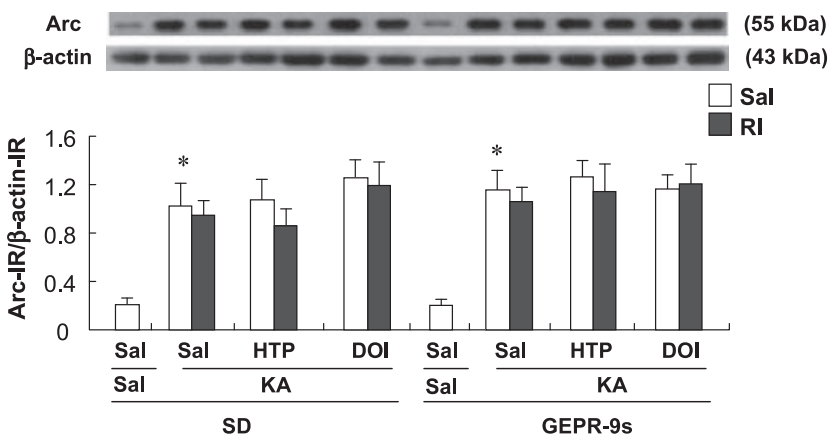

Fig. 2. Effects of ritanserin (RI, $0.5 \mathrm{mg} / \mathrm{kg}$, i.p.) on 5-hydroxytryptophan (HTP, $75 \mathrm{mg} / \mathrm{kg}$, i.p.)- and 2,5-dimethoxy-4-iodoamphetamine (DOI, $0.3 \mathrm{mg} / \mathrm{kg}$, s.c.)-mediated pharmacological responses to KA ( $8 \mathrm{mg} / \mathrm{kg}$, i.p.)-induced seizure latency (A), seizure activity (B), and hippocampal Arc-IR (C) $4 \mathrm{~h}$ after KA in SD rats and GEPR-9s. $\mathrm{Sal}=$ saline treatment. $\mathrm{M}=$ mortality (the number of dead animals / total number of animals receiving each treatment). Arc-IR = Activityregulated, cytoskeleton-associated protein immunoreactivity. Each value is the mean \pm S.E.M. of 10 animals for seizure activity and 5 animals for Arc expression. ${ }^{*} P<0.01$ vs. corresponding $\mathrm{Sal}+\mathrm{Sal}$; ${ }^{\#} P<0.05$ vs. Sal + KA-treated SD (one-way ANOVA followed by Fisher's PLSD test).

KA-induced altered WDS responses (i.e., latency and number) and hippocampal Arc protein expression via unknown mechanisms, although this agent itself can not directly affect hippocampal Arc mRNA expression (11).

In this study, hippocampal Arc-expression induced by KA with or without a serotonergic agent paralleled the number of WDS. Previous reports suggested that an increase in the central post-synaptic monoamineand glutamate-signaling activates Arc expression (12, 13). Thus, it is possible that a low level of 5-HT in neuronal synapses caused by impaired serotonergic synaptic transmission (i.e., release) may contribute to a 
decrease in KA-induced Arc expression in GEPR-9s. In contrast, serotonergic activation significantly enhanced KA-induced Arc expression under WDS phase $(1.5 \mathrm{~h}$ post-KA), suggesting that 5-HT may be involved in early responsiveness to KA.

Serotonergic agents, however, did not affect KAinduced seizures ( $4 \mathrm{~h}$ post-KA) and Arc protein expression in either strain, although Arc is assumed to be related to neural plasticity and that seizure activity could produce a differential Arc expression between GEPR-9s and SD. Therefore, it remains to be further explored.

In summary, our results suggest that KA-induced WDS is less frequent in GEPR-9s than in SD, that changes in WDS latency and Arc expression in the presence of a serotonergic agent were more pronounced in GEPR-9s than in SD, and that KA-induced altered WDS responses (i.e., an increase in latency and a decrease in number) in GEPR-9s are attributable to an abnormality in serotonergic synaptic transmission downstream of KA neuronal excitation.

\section{Acknowledgments}

This study was supported by a grant (\#2009K001253) from the Brain Research Center from the 21st Century Frontier Research Program funded by the Ministry of Science and Technology, Republic of Korea. TW Kim is involved in the BK 21 program, Korea Research Foundation. Equipment at the Institute of Pharmaceutical Science (Kangwon National University) was used for this study.

\section{References}

1 Dailey JW, Reigel CE, Mishra PK, Jobe PC. Neurobiology of seizure predisposition in the genetically epilepsy-prone rat. Epilepsy Res. 1989;3:3-17.

2 Ryu JR, Shin CY, Park KH, Jeon GS, Kim HC, WK Kim, et al. Effect of repeated seizure experiences on tyrosine hydroxylase immunoreactivities in the brain of genetically epilepsy-prone rats. Brain Res Bull. 2000;53:777-782.

3 Dursun SM, Handley SL. Similarities in the pharmacology of spontaneous and DOI-induced head-shakes suggest 5HT2A receptors are active under physiological conditions. Psychopharmacology (Berl). 1996;128:198-205.

4 Yap CY, Taylor DA. Involvement of 5-HT2 receptors in the wet-dog shake behaviour induced by 5-hydroxytryptophan in the rat. Neuropharmacology. 1983;22:801-804.

5 Tzingounis AV, Nicoll RA. Arc/Arg3.1: Linking gene expression to synaptic plasticity and memory. Neuron. 2006;52:403407.

6 Steward O, Wallace CS, Lyford GL, Worley PF. Synaptic activation causes the mRNA for the IEG Arc to localize selectively near activated postsynaptic sites on dendrites. Neuron. 1998;21:741-751.

7 Alme MN, Wibrand K, Dagestad G, Bramham CR. Chronic fluoxitine treatment induces brain region-specific upregulation of genes associated with BDNF-induced long-term potentiation. Neural Plast. 2007;2007:26496.

8 Shin EJ, Ko KH, Kim WK, Chae JS, Yen TP, Kim HJ, et al. Role of glutathione peroxidase in the ontogeny of hippocampal oxidative stress and kainate seizure sensitivity in the genetically epilepsy-prone rats. Neurochem Int. 2008;52:1134-1147.

9 Racine RJ. Modification of seizure activity by electrical stimulation. II. Motor seizure. Electroencephalogr Clin Neurophysiol. 1972;32:281-294.

10 Li L, Carter J, Gao X, Whitehead J, Tourtellotte. The neuroplasticity-associated Arc gene is a direct transcriptional target of early growth response (Egr) transcription factors. Mol Cell Biol. 2005;25:10286-10300.

11 Pei Q, Lewis L, Sprakes ME, Jones EJ, Grahame-Smith DG, Zetterstrom TSC. Serotonergic regulation of mRNA expression of Arc, an immediate early gene selectively localized at neuronal dendrites. Neuropharmacology. 2000;39:463-470.

12 Pei Q, Zetterstrom TS, Sprakes M, Tordera R, Sharp T. Antidepressant drug treatment induces Arc gene expression in the rat brain. Neuroscience. 2003;121:975-982.

13 Steward O, Worley PF. Selective targeting of newly synthesized Arc mRNA to active synapses requires NMDA receptor activation. Neuron. 2001;30:227-240. 